\title{
Models with commutative orthogonal block structure: a general condition for commutativity
}

\author{
C. Santos, C. Nunes, C. Dias \& J.T. Mexia
}

To cite this article: C. Santos, C. Nunes, C. Dias \& J.T. Mexia (2020): Models with commutative orthogonal block structure: a general condition for commutativity, Journal of Applied Statistics, DOI: 10.1080/02664763.2020.1765322

To link to this article: https://doi.org/10.1080/02664763.2020.1765322

\section{Published online: 14 May 2020.}

Submit your article to this journal $\square$

山 Article views: 31

Q View related articles ๘

View Crossmark data 


\title{
Models with commutative orthogonal block structure: a general condition for commutativity
}

\author{
C. Santos (1D) a,e , C. Nunes (1) ${ }^{b}$, C. Dias (1) ${ }^{c, e}$ and J.T. Mexia (1) d,e \\ a Department of Mathematics and Physical Sciences, Polytechnic Institute of Beja, Beja, Portugal; \\ ${ }^{b}$ Department of Mathematics and Center of Mathematics and Applications, University of Beira Interior, \\ Covilhã, Portugal; ${ }^{C}$ Polytechnic Institute of Portalegre, Portalegre, Portugal; ${ }^{\mathrm{d}}$ Department of Mathematics, \\ Faculty of Science and Technology, New University of Lisbon, Portugal; ${ }^{\mathrm{e}} \mathrm{CMA}$ - Center of Mathematics and its \\ Applications, Faculty of Science and Technology, New University of Lisbon, Portugal
}

\begin{abstract}
A linear mixed model whose variance-covariance matrix is a linear combination of known pairwise orthogonal projection matrices that add to the identity matrix, is a model with orthogonal block structure (OBS). OBS have estimators with good behavior for estimable vectors and variance components, moreover it may be interesting that the least squares estimators give the best linear unbiased estimators, for estimable vectors. We can achieve it, requiring commutativity between the orthogonal projection matrix, on the space spanned by the mean vector, and the orthogonal projection matrices involved in the expression of the variance-covariance matrix. This commutativity condition defines a more restrict class of OBS, named COBS (model with commutative orthogonal block structure). With this work we aim to present a commutativity condition, resorting to a special class of matrices, named U-matrices.
\end{abstract}

\section{ARTICLE HISTORY}

Received 17 November 2018

Accepted 30 April 2020

\section{KEYWORDS}

U-matrices; best linear unbiased estimators; mixed models; models with commutative orthogonal block structure

\section{Introduction}

Linear mixed models play an important role in the design and analysis of experiments and have a wide use in several fields.

In the framework of the design of experiments in agricultural trials, Nelder $[13,14]$ introduced models with orthogonal block structure (OBS), which are linear mixed models whose variance-covariance matrix is a linear combination of known pairwise orthogonal projection matrices (POPM) that add up to the identity matrix. OBS continue to play a central part in the theory of randomized block designs, (see [2,3]), which highlights the interest on the adequacy of the estimators, see e.g. [1,6].

OBS allow optimal estimation for variance components of blocks and contrasts of treatments [8] moreover we may be interested in that least squares estimators (LSE), giving best linear unbiased estimators (BLUE), for estimable vectors. For this purpose, we must impose a commutativity condition on OBS, as it was done in Fonseca et al. [10] when introducing 
models with commutative orthogonal block structure (COBS). COBS has been the subject of extensive research, addressing, e.g. estimation, inference and operations with models, see e.g. $[4-6,8,12,15]$.

This paper is structured as follows. A framework for models with COBS and some of their interesting results is provided in Section 2. Section 3 is dedicated to our main goal, which is to present a commutativity condition and other results enabling the obtention of BLUE. A real data application, considering an experiment with grapevines, is presented in Section 4 to illustrate the usefulness of the methodology. We conclude this work in Section 5, with some comments.

\section{Models with commutative orthogonal block structure}

To study COBS we resort to an approach based on their algebraic structure, since this leads to interesting results on the estimation of variance components and on the building up of models, see [10].

Let us consider a linear mixed model

$$
\boldsymbol{Y}=\sum_{i=0}^{w} \boldsymbol{X}_{i} \boldsymbol{\beta}_{i}
$$

where $\boldsymbol{\beta}_{0}$ is fixed and $\boldsymbol{\beta}_{1}, \ldots, \boldsymbol{\beta}_{w}$ are random vectors with null mean vectors, variancecovariance matrices $\sigma_{1}^{2} \boldsymbol{I}_{c_{1}} \ldots \sigma_{w}^{2} \boldsymbol{I}_{\mathcal{c}_{w}}$, where $c_{i}=\operatorname{rank}\left(\boldsymbol{X}_{\boldsymbol{i}}\right), i=1, \ldots, w$, and null crosscovariance matrices.

The mean vector of $\boldsymbol{Y}$ is

$$
\boldsymbol{\mu}=\boldsymbol{X}_{0} \boldsymbol{\beta}_{0}
$$

and the variance-covariance matrix is given by

$$
\boldsymbol{V}\left(\boldsymbol{\sigma}^{2}\right)=\sum_{i=1}^{w} \sigma_{i}^{2} \boldsymbol{M}_{i}
$$

where $\boldsymbol{M}_{i}=\boldsymbol{X}_{i} \boldsymbol{X}_{i}^{T}, i=1, \ldots, w$.

The space spanned by the mean vector $\boldsymbol{\mu}$ is $\Omega=R\left(\boldsymbol{X}_{0}\right)$, so the orthogonal projection matrix (OPM), on $\Omega$, is

$$
\boldsymbol{T}=\boldsymbol{X}_{0}\left(\boldsymbol{X}_{0}^{T} \boldsymbol{X}_{0}\right)^{+} \boldsymbol{X}_{0}^{T}=\boldsymbol{X}_{0} \boldsymbol{X}_{0}^{+}
$$

see e.g. [5], where+denotes the Moore-Penrose inverse.

When the matrices $\boldsymbol{M}_{1}, \ldots, \boldsymbol{M}_{w}$ commute, they generate a commutative Jordan algebra of symmetric matrices, CJAS, $A$, this is, a linear space constituted by symmetric matrices that commute and containing the squares of its matrices [11]. The CJAS, $A$ has a unique basis, its principal basis, $Q$, constituted by known pairwise orthogonal orthogonal projection matrices, POPM, $\boldsymbol{Q}_{1}, \ldots, \mathbf{Q}_{m}$, see [17]. Thus the matrices $\boldsymbol{M}_{i}, i=1, \ldots, w$, are linear 
combinations of the matrices of the principal basis of the CJAS, which means that

$$
\boldsymbol{M}_{i}=\sum_{j=1}^{m} b_{i, j} \boldsymbol{Q}_{j} .
$$

Considering $\gamma_{j}=\sum_{i=1}^{w} b_{i, j} \sigma_{i}^{2}, j=1, \ldots, m$, the canonical variance components, the variance-covariance matrix of $\boldsymbol{Y}$ will take the form

$$
\boldsymbol{V}=\sum_{j=1}^{m} \gamma_{j} \boldsymbol{Q}_{j}
$$

When $\sum_{i=1}^{w} \boldsymbol{M}_{i}$, belonging to $A$, is invertible, $A$ is a complete CJAS and the matrices of its principal basis add up to the identity matrix, i.e.

$$
\sum_{j=1}^{m} \boldsymbol{Q}_{j}=\boldsymbol{I}_{n},
$$

and model (1) is a model with OBS.

When dealing with OBS, inference usually involves orthogonal projections on the range spaces of the matrices $\boldsymbol{Q}_{j}, j=1, \ldots, m$, which is somewhat complex due to the combination of estimators obtained from different projections, see e.g. [4]. Imposing a commutativity condition on the OPM on the space spanned by the mean vector, $\boldsymbol{T}$, and the POPM $\boldsymbol{Q}_{j}$, $j=1, \ldots, m$, leads to a special class of OBS, those of models with COBS, see [10]. For this class of models we do not have the difficulty associated with orthogonal projections mentioned above, allowing, additionally, the least square estimators, for estimable vectors, to be UBLUE. According to the version of the Gauss-Markov theorem in [18], UBLUE are BLUE whatever the variance components.

\section{Generalizing the commutativity condition}

Assuming the rows of matrix $\boldsymbol{X}_{0}$ to correspond to the sets of levels of the fixed effects factors, the mean values of the observations will be determined by those sets. Let us consider that there are $\dot{n}$ sets of levels associated to $r_{1}, \ldots, r_{\dot{n}}$, contiguous rows of $\boldsymbol{X}_{0}$. If the components of $\boldsymbol{\beta}_{0}, \beta_{0,1}, \ldots, \beta_{0, \dot{n}}$, are the corresponding mean values, we can reorder the observations to have the block diagonal matrix

$$
\boldsymbol{X}_{0}=D\left(1_{r_{1}}, \ldots, 1_{r_{\dot{n}}}\right),
$$

where $1_{r_{l}}$, corresponds to the vector with all $r_{l}$ components equal to $1, l=1, \ldots, \dot{n}$. So, the orthogonal projection matrix on the space spanned by the mean vector, is given by

$$
\boldsymbol{T}=D\left(\frac{1}{r_{1}} \boldsymbol{J}_{r_{1}}, \ldots, \frac{1}{r_{\dot{n}}} \boldsymbol{J}_{r_{\dot{n}}}\right)
$$

where $\boldsymbol{J}_{r_{l}}=1_{r_{l}} 1_{r_{l}}^{T}, l=1, \ldots, \dot{n}$. 
The fundamental partition of $\boldsymbol{Y}$ will be constituted by the sub-vectors $\boldsymbol{Y}_{1}, \ldots, \boldsymbol{Y}_{\dot{n}}$, corresponding to the $\dot{n}$ sets of the levels of the fixed effects factors, see [16]. Then the variancecovariance matrix can be defined by

$$
\boldsymbol{V}=\left[\begin{array}{ccc}
\boldsymbol{V}_{1,1} & \ldots & \boldsymbol{V}_{1, \dot{n}} \\
\vdots & & \vdots \\
\boldsymbol{V}_{\dot{n}, 1} & \ldots & \boldsymbol{V}_{\dot{n}, \dot{n}}
\end{array}\right],
$$

with $V_{l, l}$ the variance-covariance matrix of $Y_{l}, l=1, \ldots, \dot{n}$, and $V_{l, h}$ the cross-covariance matrix of $\boldsymbol{Y}_{l}$ and $\boldsymbol{Y}_{h}, l \neq h$.

When $\boldsymbol{T}$, the OPM on the space spanned by the mean vector $\boldsymbol{\mu}$, commutes with the POPM $\boldsymbol{Q}_{j}, j=1, \ldots, m$, the OPM also commutes with the variance-covariance matrix of $\boldsymbol{Y}, \boldsymbol{V}$.

From (8) and (9) we have

$$
\boldsymbol{T} \boldsymbol{V}=\left[\begin{array}{ccc}
\frac{1}{r_{1}} \boldsymbol{J}_{r_{1}} \boldsymbol{V}_{1,1} & \ldots & \frac{1}{r_{1}} \boldsymbol{J}_{r_{1}} \boldsymbol{V}_{1, \dot{n}} \\
\vdots & & \vdots \\
\frac{1}{r_{\dot{n}}} \boldsymbol{J}_{r_{\dot{n}}} \boldsymbol{V}_{\dot{n}, 1} & \cdots & \frac{1}{r_{\dot{n}}} \boldsymbol{J}_{r_{\dot{n}}} \boldsymbol{V}_{\dot{n}, \dot{n}}
\end{array}\right]
$$

and

$$
\boldsymbol{V T}=\left[\begin{array}{ccc}
\boldsymbol{V}_{1,1} \frac{1}{r_{1}} \boldsymbol{J}_{r_{1}} & \ldots & \boldsymbol{V}_{1, \dot{n}} \frac{1}{r_{\dot{n}}} \boldsymbol{J}_{r_{\dot{n}}} \\
\vdots & & \vdots \\
\boldsymbol{V}_{\dot{n}, 1} \frac{1}{r_{1}} \boldsymbol{J}_{r_{1}} & \ldots & \boldsymbol{V}_{\dot{n}, \dot{n}} \frac{1}{r_{\dot{n}}} \boldsymbol{J}_{r_{\dot{n}}}
\end{array}\right]
$$

So, the matrices $\boldsymbol{T}$ and $\boldsymbol{V}$ commute if and only if

$$
\left\{\begin{array}{cccc}
\frac{1}{r_{1}} \boldsymbol{J}_{r_{1}} \boldsymbol{V}_{1,1}=\boldsymbol{V}_{1,1} \frac{1}{r_{1}} \boldsymbol{J}_{r_{1}} & \ldots & \frac{1}{r_{1}} \boldsymbol{J}_{r_{1}} \boldsymbol{V}_{1, \dot{n}}=\boldsymbol{V}_{1, \dot{n}} \frac{1}{r_{\dot{n}}} \boldsymbol{J}_{r_{\dot{n}}} \\
\vdots & & \vdots \\
\frac{1}{r_{\dot{n}}} \boldsymbol{J}_{r_{\dot{n}}} \boldsymbol{V}_{\dot{n}, 1}=\boldsymbol{V}_{\dot{n}, 1} \frac{1}{r_{1}} \boldsymbol{J}_{r_{1}} & \ldots & \frac{1}{r_{\dot{n}}} \boldsymbol{J}_{r_{\dot{n}}} \boldsymbol{V}_{\dot{n}, \dot{n}}=\boldsymbol{V}_{\dot{n}, \dot{n}} \frac{1}{r_{\dot{n}}} \boldsymbol{J}_{r_{\dot{n}}}
\end{array} .\right.
$$

These equalities imply that we must have

$$
r_{1}=\ldots=r_{\dot{n}}=r
$$

and equalities (12) may be condensed into

$$
\boldsymbol{J}_{r} \boldsymbol{V}_{l, h}=\boldsymbol{V}_{l, h} \boldsymbol{J}_{r}, l, h=1, \ldots, \dot{n} .
$$

Now, given a matrix

$$
\boldsymbol{U}=\left[\begin{array}{ccc}
u_{1,1} & \ldots & u_{1, r} \\
\vdots & & \vdots \\
u_{r, 1} & \ldots & u_{r, r}
\end{array}\right]
$$


we have

$$
\boldsymbol{J}_{r} \boldsymbol{U}=\left[\begin{array}{ccc}
\sum_{l=1}^{r} u_{l, 1} & \ldots & \sum_{l=1}^{r} u_{l, r} \\
\vdots & & \vdots \\
\sum_{l=1}^{r} u_{l, 1} & \ldots & \sum_{l=1}^{r} u_{l, r}
\end{array}\right]
$$

and

$$
\boldsymbol{U J}_{r}=\left[\begin{array}{ccc}
\sum_{h=1}^{r} u_{1, h} & \ldots & \sum_{h=1}^{r} u_{1, h} \\
\vdots & & \vdots \\
\sum_{h=1}^{r} u_{r, h} & \ldots & \sum_{h=1}^{r} u_{r, h}
\end{array}\right] \text {. }
$$

So, to have the equality

$$
J_{r} U=U J_{r}
$$

we must have

$$
\sum_{l^{\prime}=1}^{r} u_{l^{\prime}, h}=\sum_{h^{\prime}=1}^{r} u_{l, h^{\prime}}=\frac{\bar{u}}{r}, l, h=1, \ldots, r,
$$

with $\bar{u}=\sum_{l^{\prime}=1}^{r} \sum_{h^{\prime}=1}^{r} u_{l^{\prime}, h^{\prime}}$, which means that the sums of the elements in any row or column of matrix $\boldsymbol{U}$ are equal. Thus, matrix $\boldsymbol{U}$ is called a $U$-matrix, see [16].

Going back to the product of matrices $V$ and $T$, we see that these matrices commute if and only if the sub-matrices $V_{l, h}, l, h=1, \ldots, m$, are U-matrices. We thus have the following result.

Proposition 1: For the LSE of $\boldsymbol{\beta}_{0}$ be UBLUE it is necessary and sufficient that $r_{1}=\ldots=$ $r_{\dot{n}}=r$ and the sub-matrices $V_{l, h}, l, h=1, \ldots, \dot{n}$ be U-matrices.

Since we have

$$
\boldsymbol{X}_{0}=D\left(1_{r}, \ldots, 1_{r}\right)=\boldsymbol{I}_{m} \otimes 1_{r}
$$

where $\otimes$ denotes the Kronecker matrices product, and taking $\dot{n}=m$ we also have

$$
\left(\boldsymbol{X}_{0}^{T} \boldsymbol{X}_{0}\right)^{-1}=\frac{1}{r} \boldsymbol{I}_{m}
$$

and so $\left(\boldsymbol{X}_{0}{ }^{T} \boldsymbol{X}_{0}\right)^{-1} \boldsymbol{X}_{0}{ }^{T}=(1 / r) D\left(1_{r}, \ldots, 1_{r}\right)$. Thus, the components of

$$
\widetilde{\boldsymbol{\beta}_{0}}=\left(\boldsymbol{X}_{0}^{T} \boldsymbol{X}_{0}\right)^{-1} \boldsymbol{X}_{0}^{T}\left(\boldsymbol{Y}_{1}^{T} \ldots \boldsymbol{Y}_{m}^{T}\right)^{T}
$$

will be the mean values $y_{0,1}, \ldots, y_{0, m}$ of the components of the sub-vectors $\boldsymbol{Y}_{1}, \ldots, \boldsymbol{Y}_{m}$. 
We are thus led to replace $\boldsymbol{\beta}_{0}$ and $\tilde{\boldsymbol{\beta}_{0}}$ by $\boldsymbol{\mu}_{0}=\left(\boldsymbol{\mu}_{0,1}, \ldots, \boldsymbol{\mu}_{0, m}\right)$ and $\tilde{\boldsymbol{\mu}}_{0}=$ $\left(\tilde{\boldsymbol{\mu}}_{0,1}, \ldots, \tilde{\boldsymbol{\mu}}_{0, m}\right)$, respectively, which enables us to consider other parametrizations, taking

$$
\boldsymbol{\mu}_{0}=\boldsymbol{G} \boldsymbol{\beta}_{0}
$$

where $\boldsymbol{G}$ will have linearly independent column vectors. Then $\tilde{\boldsymbol{\mu}}_{0}$ will be the matrix of sub-vectors means and, since

$$
\boldsymbol{\beta}_{0}=\boldsymbol{G}^{+} \boldsymbol{\mu}_{0}
$$

we have the estimator

$$
\widetilde{\boldsymbol{\beta}}_{0}=\boldsymbol{G}^{+} \tilde{\boldsymbol{\mu}}_{0} .
$$

We also have the following proposition.

Proposition 2: The estimator $\widetilde{\boldsymbol{\beta}_{0}}$ is UBLUE.

Proof: Let $\boldsymbol{\beta}_{0}^{*}$ be another unbiased estimator of $\boldsymbol{\beta}_{0}$. Then, for $\boldsymbol{c}^{T} \boldsymbol{\beta}_{0}$ we have the unbiased estimator $\boldsymbol{c}^{T} \boldsymbol{\beta}_{0}=\boldsymbol{a}^{T} \tilde{\boldsymbol{\mu}}_{0}$ with $\boldsymbol{a}^{T}=\boldsymbol{c}^{T} \boldsymbol{G}^{+}$and $\boldsymbol{c}^{T} \boldsymbol{\beta}_{0}^{*}=\boldsymbol{a}^{T} \boldsymbol{\mu}_{0}^{*}$, with $\boldsymbol{\mu}_{0}^{*}=\boldsymbol{G} \boldsymbol{\beta}_{0}^{*}$. Since $\boldsymbol{\mu}_{0}^{*}$ is an unbiased estimator of $\boldsymbol{\mu}_{0}$, and $\tilde{\boldsymbol{\mu}}_{0}$ is UBLUE for $\boldsymbol{\mu}_{0}$, we have $\operatorname{Var}\left(\boldsymbol{c}^{T} \widetilde{\boldsymbol{\beta}}_{0}\right) \leq \operatorname{Var}\left(\boldsymbol{c}^{T} \boldsymbol{\beta}_{0}^{*}\right)$ whatever the variance components. Given that $\boldsymbol{c}$ is arbitrary, $\widetilde{\boldsymbol{\beta}}_{0}$ is BLUE. Since this holds for all variance components $\widetilde{\boldsymbol{\beta}_{0}}$ is UBLUE.

Similarly, we may consider

$$
\lambda=\boldsymbol{U} \boldsymbol{\mu}_{0},
$$

with the column vectors of $\boldsymbol{U}$ linearly independent. We now have the result.

Proposition 3: $\tilde{\lambda}=\boldsymbol{U} \tilde{\boldsymbol{\mu}}_{0}$ will be UBLUE for $\lambda$.

Proof: Since the column vectors of $\boldsymbol{U}$ are linearly independent we have $\boldsymbol{\mu}_{0}=\boldsymbol{U}^{+} \boldsymbol{\lambda}$ and $\tilde{\boldsymbol{\mu}}_{0}=\boldsymbol{U}^{+} \tilde{\boldsymbol{\lambda}}$. Given $\lambda^{*}$ an unbiased estimator of $\lambda, \boldsymbol{\mu}_{0}{ }^{*}=\boldsymbol{U}^{+} \lambda^{*}$ will be an unbiased estimator of $\lambda$ since its mean vector will be $\boldsymbol{U}^{+} \boldsymbol{U} \boldsymbol{\mu}_{0}=\boldsymbol{\mu}_{0}$. We also have $\lambda^{*}=\boldsymbol{U} \boldsymbol{\mu}_{0}{ }^{*}$. Moreover, for $\boldsymbol{c}^{T} \boldsymbol{\lambda}$ we have the unbiased estimators $\boldsymbol{c}^{T} \tilde{\boldsymbol{\lambda}}=\boldsymbol{c}^{T} \boldsymbol{U} \tilde{\boldsymbol{\mu}}_{0}=\left(\boldsymbol{U}^{T} \boldsymbol{c}\right)^{T} \tilde{\boldsymbol{\mu}}_{0}$ and $\boldsymbol{c}^{T} \boldsymbol{\lambda}^{*}=$ $\boldsymbol{c}^{T} \boldsymbol{U} \boldsymbol{\mu}_{0}{ }^{*}=\left(\boldsymbol{U}^{T} \boldsymbol{c}\right)^{T} \boldsymbol{\mu}_{0}{ }^{*}$. Since $\tilde{\boldsymbol{\mu}}_{0}$ is BLUE for $\boldsymbol{\mu}_{0}$, we have $\operatorname{Var}\left(\boldsymbol{c}^{T} \tilde{\boldsymbol{\lambda}}\right) \leq \operatorname{Var}\left(\boldsymbol{c}^{T} \boldsymbol{\lambda}^{*}\right)$, whatever $c$, which shows that $\tilde{\lambda}$ is BLUE. Since this holds for all variance components $\tilde{\lambda}$ is UBLUE.

\section{An application}

Let's consider an experiment with 'Touriga Nacional' grapevine and two fixed effects factors:

- Location (in the experiment), with three levels;

- Origin, with two levels.

These two factors cross. Given the great number of clones, some ones were randomly chosen and considered as the levels of a random effects factor nested in the factor Origin. 
Table 1. Production in $\mathrm{Kg}$.

\begin{tabular}{cccccccc}
\hline & \multicolumn{3}{c}{ ORIGIN 1 } & & \multicolumn{3}{c}{ ORIGIN 2 } \\
\cline { 2 - 5 } \cline { 6 - 8 } LOCATION & Clone 1 & Clone 2 & Clone 3 & & Clone 1 & Clone 2 & Clone 3 \\
\hline 1 & 3,00 & 1,00 & 1,10 & & 1,75 & 1,10 & 1,05 \\
& 1,85 & 1,10 & 1,50 & & 3,50 & 1,05 & 1,25 \\
& 0,75 & 1,00 & 1,80 & & 2,50 & 0,50 & 2,00 \\
& 1,35 & 1,60 & 1,45 & & 2,00 & 1,05 & 1,50 \\
& 1,45 & 1,50 & 1,25 & & 0,65 & 1,25 & 2,10 \\
& 1,80 & 1,60 & 0,85 & & 2,00 & 1,20 & 1,00 \\
& 0,70 & 1,75 & 0,65 & & 3,00 & 1,35 & 2,70 \\
& 2,50 & 0,50 & 0,55 & & 2,55 & 1,20 & 2,15 \\
& 1,70 & 1,35 & 0,90 & & 3,00 & 0,30 & 2,10 \\
& 0,40 & 1,10 & 0,09 & & 2,65 & 2,50 & 2,70 \\
& 1,05 & 0,75 & 0,90 & & 1,60 & 1,05 & 1,60 \\
& 1,50 & 0,65 & 0,90 & & 3,05 & 1,95 & 1,10 \\
& 1,15 & 0,90 & 0,55 & & 0,25 & 2,00 & 2,05 \\
& 0,85 & 0,85 & 0,70 & & 1,66 & 2,20 & 1,50 \\
& 1,15 & 1,05 & 0,35 & & 2,65 & 2,35 & 3,00 \\
\hline
\end{tabular}

For each origin, three clones were randomly chosen. Lastly five grapevines were considered for each clone in each location. This experiment was analyzed, see [7,9], using its algebraic structure, namely using CJA. For completeness sake we now apply our approach.

We have $\dot{n}=3 \times 2=6$ sub-vectors each with $r=3 \times 5=15$ observations. These vectors are presented in Table 1.

With $\mu$ : the general mean; $\alpha_{i}$ : the effect of the $i$-th location, $i=1,2,3 ; \beta_{j}$ : the effect of the $j$-th origin, $j=1,2 ; \gamma_{i, j}$ : the interaction between the $i$-th location and the $j$-th origin, $i=1,2,3, j=1,2 ; a_{l, j}$ : the random effect of the $l$-th clone of the $j$-th origin, $l=1,2,3 ; j=$ 1,2 ; we have, for the sub-vectors, the model equation

$$
\boldsymbol{Y}_{i, j}=\left(\mu+\alpha_{i}+\beta_{j}+\gamma_{i, j}\right) \mathbf{1}_{15}+\left[\begin{array}{l}
a_{1, j} \\
a_{2, j} \\
a_{3, j}
\end{array}\right] \otimes \mathbf{1}_{5}+\boldsymbol{e}_{i, j} ; i=1,2,3 ; j=1,2,
$$

where the $\boldsymbol{e}_{i, j}, i=1,2,3, j=1,2$, will be normal with null mean vector and variancecovariance matrix $\sigma_{l}^{2} \boldsymbol{I}_{15}$ independent from the vector $\boldsymbol{a}_{j}$, with components $\left(a_{1, j}, a_{2, j}, a_{3, j}\right)$, $j=1,2$, which will be normal with null mean vector and covariance matrix $\sigma_{a}^{2} \boldsymbol{I}_{3}$. We can order these sub-vectors using the index $l=2(i-1)+j ; i=1,2,3 ; j=1,2$.

It is straightforward to obtain the covariance and cross-covariance matrices of the subvectors. We thus get

$$
\begin{gathered}
V_{1,1}=V_{2,2}=V_{3,3}=V_{4,4}=V_{5,5}=V_{6,6}=\sigma_{a}^{2} I_{3} \otimes J_{5}+\sigma_{l}^{2} I_{15} \\
V_{1,2}=V_{2,1}=V_{3,4}=V_{4,3}=V_{5,6}=V_{6,5}=0_{15,15} \\
V_{1,3}=V_{3,1}=V_{1,5}=V_{5,1}=V_{2,4}=V_{4,2}=\sigma_{a}^{2} I_{3} \otimes I_{5} \\
V_{1,4}=V_{4,1}=V_{2,3}=V_{3,2}=V_{1,6}=V_{6,1}=0_{15,15} \\
V_{1,6}=V_{6,1}=V_{2,5}=V_{5,2}=V_{3,6}=V_{6,3}=0_{15,15}
\end{gathered}
$$


It is easy to see that all these matrices are U-matrices. Thus, the LSE estimator of the vector $\boldsymbol{\mu}_{0}$ with components

$$
\mu_{i, j}=\mu+\alpha_{i}+\beta_{j}+\gamma_{i, j} ; i=1,2,3 ; j=1,2
$$

will be UBLUE.

In this application we will focus in the LSE for $\boldsymbol{\beta}$. The ANOVA analysis is standard. It is interesting to point out that, since

$$
\boldsymbol{X}_{0}=D\left(1_{r}, \ldots, 1_{r}\right)=\boldsymbol{I}_{m} \otimes 1_{r}
$$

we have

$$
\left(\boldsymbol{X}_{0}^{T} \boldsymbol{X}_{0}\right)^{-1} \boldsymbol{X}_{0}^{T}=\frac{1}{r} D\left(1_{r}, \ldots, 1_{r}\right)=\frac{1}{r} \boldsymbol{I}_{m} \otimes 1_{r} .
$$

Thus, the components of $\widetilde{\boldsymbol{\beta}_{0}}$ will be the means of the components of sub-vectors. We will represent those means by $\tilde{\mu}_{i, j}, i=1,2,3 ; j=1,2$. Taking

$$
\left\{\begin{array}{l}
\tilde{\mu}_{i, .}=\frac{1}{2}\left(\tilde{\mu}_{i, 1}+\tilde{\mu}_{i, 2}\right), i=1,2,3 \\
\tilde{\mu}_{., j}=\frac{1}{3}\left(\tilde{\mu}_{1, j}+\tilde{\mu}_{2, j}+\tilde{\mu}_{3, j}\right), j=1,2 \\
\tilde{\mu}_{., .}=\frac{1}{6} \sum_{i=1}^{3} \sum_{j=1}^{2} \tilde{\mu}_{i, j}
\end{array}\right.
$$

we get the estimators

$$
\left\{\begin{array}{l}
\tilde{\alpha}_{i}=\tilde{\mu}_{i, .}-\tilde{\mu}_{., .}, i=1,2,3 \\
\tilde{\beta}_{j}=\tilde{\mu}_{., j}-\tilde{\mu}_{., .}, j=1,2 \\
\tilde{\gamma}_{i, j}=\tilde{\mu}_{i, j}-\tilde{\mu}_{i, .}-\tilde{\mu}_{., j}+\tilde{\mu}_{., .}, i=1,2,3 ; j=1,2
\end{array} .\right.
$$

According to Proposition 1 these estimators will be UBLUE.

Besides this, using software $\mathrm{R}$, we carried out a standard ANOVA whose main results are presented in Table 2.

From the results presented in Table 2, we conclude that interaction between the fixed effects factors (Location and Origin) and Location are significant.

\section{Final comments}

The use of linear mixed models is suitable for correlated data due to, for example, repeated measurements. From Nelder's work emerged a particular class of linear mixed models, named OBS, that took a central role in the theory of randomized block designs, giving rise to several lines of research. As a relevant step towards the adequacy of the estimators came a special class of OBS, called COBS, which allows the estimation of relevant parameters to be optimized. COBS are based on commutativity between $T$, the OPM on the space spanned by the mean vector, and the POPM $\boldsymbol{Q}_{j}, j=1, \ldots, m$. The commutativity condition we presented is easy to verify and guaranties UBLUE estimators, obtained through least squares, for the coefficients vector and estimable vectors. Thus, we consider that our aims were attained. 
Table 2. Model summary and ANOVA table.

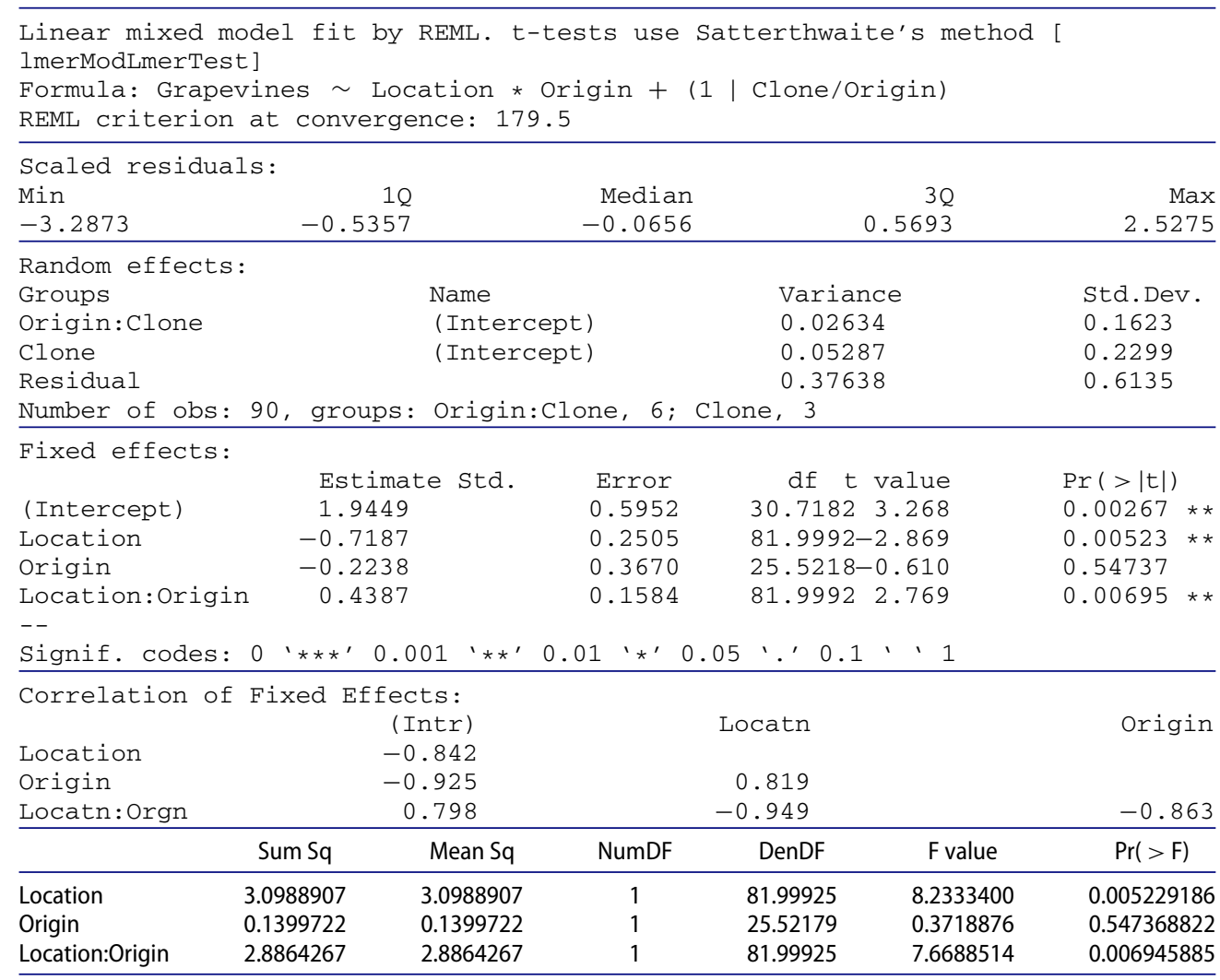

\section{Acknowledgements}

We thank the referees for their valuable comments on our manuscript which led to significant improvement of this work.

\section{Disclosure statement}

No potential conflict of interest was reported by the author(s).

\section{Funding}

This work was partially supported by national founds of FCT-Foundation for Science and Technology under UID/MAT/00297/2019 and UID/MAT/00212/2019.

\section{ORCID}

C. Santos (i) http://orcid.org/0000-0002-0077-1249

C. Nunes (1D) http://orcid.org/0000-0003-0167-4851

C. Dias (D) http://orcid.org/0000-0001-6350-5610

J.T. Mexia (iD http://orcid.org/0000-0001-8620-0721 


\section{References}

[1] R.A. Bailey, S.S. Ferreira, D. Ferreira, and C. Nunes, Estimability of variance components when all model matrices commute. Linear. Algebra. Appl. 492 (2016), pp. 144-160.

[2] T. Calinski and S. Kageyama, Block Designs: A Randomization Approach, Vol. I: Analysis, Lecture Note in Statistics, 150, Springer-Verlag, New York, 2000.

[3] T. Calinski and S. Kageyama, Block Designs: A Randomization Approach, Vol. II: Design, Lecture Note in Statistics, 170, Springer-Verlag, New York, 2003.

[4] F. Carvalho, J.T. Mexia, and M. Oliveira, Estimation in models with commutative orthogonal block structure. J. Stat. Theory. Pract. 3 (2009), pp. 523-533.

[5] F. Carvalho, J.T. Mexia, and C. Santos, Commutative orthogonal block structure and error orthogonal models. Electron. J. Linear Algebra 25 (2013), pp. 119-128.

[6] F. Carvalho, J.T. Mexia, C. Santos, and C. Nunes, Inference for types and structured families of commutative orthogonal block structures. Metrika 78 (2015), pp. 337-372.

[7] S. S. Ferreira, Inferência para Modelos Ortogonais com Segregação, Unpublished $\mathrm{PhD}$ thesis, University of Beira Interior, 2006.

[8] S. Ferreira, D. Ferreira, C. Nunes, and J.T. Mexia, Estimation of variance components in linear mixed models with commutative orthogonal block structure. Rev. Colomb. Estad. 36 (2013), pp. 261-271.

[9] S.S. Ferreira and J.T. Mexia, Alternative analysis of an experiment on grapevines. Colloq. Biometryczne 34 (2004), pp. 89-95.

[10] M. Fonseca, J.T. Mexia, and R. Zmyslony, Inference in normal models with commutative orthogonal block structure. Acta Comment. Univ. Tartu. Math. 12 (2008), pp. 3-16.

[11] P. Jordan, J. Von Neumann, and E. Wigner, On the algebraic generalization of the quantum mechanical formalism. Ann. Math. 36 (1934), pp. 26-64.

[12] J.T. Mexia, R. Vaquinhas, M. Fonseca, and R. Zmyslony, COBS: Segregation, matching, crossing and nesting. Latest Trends and Applied Mathematics, simulation, Modelling, 4-th International Conference on Applied Mathematics, Simulation, Modelling (ASM'10), 2010, pp. 249-255.

[13] J.A. Nelder, The analysis of randomized experiments with orthogonal block structure I. block structure and the null analysis of variance. Proc. R. Soc. Lond. Ser. A Math. Phys. Eng. Sci. 283 (1965), pp. 147-162.

[14] J.A. Nelder, The analysis of randomized experiments with orthogonal block structure II. Treatment structure and the general analysis of variance. Proc. R. Soc. Lond. Ser. A Math. Phys. Eng. Sci. 283 (1965), pp. 163-178.

[15] C. Santos, Error orthogonal models: structure, operations and inference, $\mathrm{PhD}$ thesis, University of Beira Interior, 2012.

[16] C. Santos, C. Nunes, and J.T. Mexia, OBS, COBS and mixed models associated to commutative Jordan Algebra. Bulletin of the ISI, LXII, Proceedings of 56th session of the International Statistical Institute. Lisbon, 2008, pp. 3271-3274.

[17] J. Seely, Quadratic subspaces and completeness. Ann. Math. Statist. 42 (1971), pp. 710-721.

[18] R. Zmyślony, A characterization of best linear unbiased estimators in the general linear model. Math. Stat. Probab. Theory 2 (1978), pp. 365-373. 